\title{
One-relator groups and proper 3-realizability
}

\author{
Manuel Cárdenas, Francisco F. Lasheras, Antonio Quintero \\ and Dušan Repovš
}

\begin{abstract}
How different is the universal cover of a given finite 2-complex from a 3-manifold (from the proper homotopy viewpoint)? Regarding this question, we recall that a finitely presented group $G$ is said to be properly 3-realizable if there exists a compact 2-polyhedron $K$ with $\pi_{1}(K) \cong G$ whose universal cover $\tilde{K}$ has the proper homotopy type of a PL 3-manifold (with boundary). In this paper, we study the asymptotic behavior of finitely generated one-relator groups and show that those having finitely many ends are properly 3-realizable, by describing what the fundamental pro-group looks like, showing a property of one-relator groups which is stronger than the QSF property of Brick (from the proper homotopy viewpoint) and giving an alternative proof of the fact that one-relator groups are semistable at infinity.
\end{abstract}

\section{Introduction}

The following question was formulated in [22] for an arbitrary finitely presented group $G$ : does there exist a compact 2 -polyhedron $K$ with $\pi_{1}(K) \cong G$ whose universal cover $\tilde{K}$ is proper homotopy equivalent to a 3-manifold? If so, the group $G$ is said to be properly 3-realizable. Recall that two spaces are said to be proper homotopy equivalent if they are homotopy equivalent and all homotopies involved are proper maps, i.e., they have the property that the inverse image of every compact subset is compact. It is a fact that one can get the above universal cover $\tilde{K}$ of $K$ proper homotopy equivalent to a 4-manifold, as one can take $K$ to be the Cayley complex associated

2000 Mathematics Subject Classification: Primary: 57M07; Secondary: 57M10, 57M20. Keywords: Proper homotopy equivalence, polyhedron, one-relator group, proper 3-realizability, end of group. 
to a (finite) group presentation of $G$, which can easily be embedded in $\mathbb{R}^{4}$. Moreover, it is known that the proper homotopy type of any locally finite 2-dimensional $C W$-complex can be represented by a subpolyhedron in $\mathbb{R}^{4}$ (see [4]). The question whether or not one can do better than this, i.e., whether or not a given finitely presented group $G$ is properly 3-realizable, is of interest as it has implications in the theory of cohomology of groups: if $G$ is properly 3-realizable then for some (equivalently any) compact 2-polyhedron $K$ with $\pi_{1}(K) \cong G$ the group $H_{c}^{2}(\tilde{K} ; \mathbb{Z})$ is free abelian (by manifold duality arguments), and hence so is $H^{2}(G ; \mathbb{Z} G)$ (see [18]). It is a long standing conjecture (attributed to Hopf) that $H^{2}(G ; \mathbb{Z} G)$ is free abelian for every finitely presented group $G$. Observe that there are examples of locally compact, simply connected 2-polyhedra $X$ for which $H_{c}^{2}(X ; \mathbb{Z})$ is not free abelian, but they are known not to be covering spaces of compact polyhedra (see [4, 22]). There are several results in the literature regarding properly 3-realizable groups (see $[1,5,7,8,23]$ ). An example of a non-3-manifold group which has this property is already pointed out in [22]. It is worth mentioning that we have recently found that there are groups which are 1-ended and semistable at infinity but not properly 3 -realizable (see $[6,13])$. The main result of the present paper is:

Theorem 1.1. Every finitely generated one-relator group $G$ with finitely many ends is properly 3-realizable.

Recall that, given a compact 2-polyhedron $K$ with $\pi_{1}(K) \cong G$ and $\tilde{K}$ as its universal cover, the number of ends of $G$ equals the number of ends of $\tilde{K}$ which in turn equals $0,1,2$ or $\infty$ (see $[17,31]$ ).

Proof of Theorem 1.1. Given a one-relator group $G$ and a presentation $P$ of $G$ with a single defining relation, it is shown in Proposition 2.7 below that we can alter (within its proper homotopy type) the universal cover $\tilde{K}_{P}$ of the standard 2-complex $K_{P}$ associated to this presentation so as to get a new 2-complex $\widehat{K}_{P}$ together with a filtration $\widehat{C}_{1} \subset \widehat{C}_{2} \subset \cdots \subset \widehat{K}_{P}$ of compact simply connected subcomplexes such that (for any given base ray in $\left.\widehat{K}_{P}\right)$ the tower of groups, pro $-\pi_{1}\left(\widehat{K}_{P}\right)$,

$$
\{1\} \leftarrow \pi_{1}\left(\widehat{K}_{P}-\operatorname{int}\left(\widehat{C}_{1}\right)\right) \leftarrow \pi_{1}\left(\widehat{K}_{P}-\operatorname{int}\left(\widehat{C}_{2}\right)\right) \leftarrow \cdots
$$

is a telescopic tower, i.e., it is a tower of finitely generated free groups of increasing bases where the bonding maps are projections (see $\S 5$ ). Thus, pro- $\pi_{1}\left(\tilde{K}_{P}\right)$ (and hence the fundamental pro-group of $G$ ) is also of that type, up to pro-isomorphism. In the 1-ended case it is known that a group with such a fundamental pro-group is properly 3-realizable (see [23, Thm.1.2]). Note that we are already done in the 0 -ended or 2 -ended case as the 0 -ended 
case corresponds to finite cyclic groups, and in the 2-ended case we only have to deal with the group $\mathbb{Z}$ of integers, which is the only one-relator group having $\mathbb{Z}$ as a subgroup of finite index. See also ([1], Cor. 1.2).

Remark 1.2. Observe that the proof of Theorem 1.1 given above shows that the group $G$ is semistable at infinity (cf. [30]). See $§ 5$.

It is worth noting that if $P$ is any finite group presentation of $G$ with a single defining relation and $K_{P}$ is the standard 2-complex (with a single vertex and a single 2-cell) associated to this presentation, then Theorem 1.1 together with ([1], Prop. 1.3) yields that the universal cover of $K_{P} \vee S^{2}$ is proper homotopy equivalent to a 3 -manifold. In fact, we conjecture that we may disregard the 2-sphere, having the universal cover of $K_{P}$ proper homotopy equivalent to a 3-manifold itself.

Corollary 1.3. Every torsion-free finitely generated one-relator group $G$ is properly 3-realizable.

Proof. Observe that, in general, if a group has infinitely many ends then Stallings' theorem tells us that it splits as an amalgamated free product or an HNN-extension over a finite group (see [31, 17]), and Dunwoody's accessibility result [11] shows that the process of further factorization of the group in this way must terminate after a finite number of steps, and each of the factors can have at most one end. In the torsion-free case, the above translates into a decomposition of $G$ into a free product of a free group with a one-relator group, the latter having at most one end. The conclusion now follows from Theorem 1.1 and the fact that free groups are properly 3-realizable, and free products of properly 3-realizable groups are again properly 3 -realizable (see ([1], Thm. 1.4)).

On the other hand, a conjecture of the following type was stated in [14]: if a torsion-free one relator group decomposes as a certain amalgamated free product $A *_{C} B$ over a free group $C$, then each of the factors $A$ and $B$ must be either a free group or a one-relator group. In fact, it was proved in [14] that this is so from the homology viewpoint. We hereby pose the following conjecture:

Conjecture 1.4. If a one-relator group with torsion decomposes as an amalgamated free product $A *_{C} B$ (resp. an $H N N$-extension $A *_{C}$ ) over a finite group $C$ (which is necessarily cyclic, see [21, 25]), then each of the factors $A$ and $B$ (resp. the base group A) must be either a one-relator group or a free product of one-relator groups (with torsion).

Observe that if this conjecture is true then, using the results of Stallings and Dunwoody (as in Corollary 1.2), the problem of showing that all finitely generated one-relator groups are properly 3-realizable can be reduced 
to the 1-ended case, by ([1, Thm.1.4]), and hence completely solved by Theorem 1.1. Thus, we conjecture the following:

Conjecture 1.5. The "finitely many ended" hypothesis in Theorem 1.1 can be omitted.

\section{One-relator groups and their structure at infinity}

The purpose of this section is to obtain some asymptotic properties of onerelator groups, which will be essential for our proof of Theorem 1.1. From now on, all complexes will be assumed to be PL CW-complexes (in the sense of $([20], \S 1.4))$.

Given a finitely presented group $G$ and a finite 2-dimensional CW-complex $X$ with $\pi_{1}(X) \cong G$, we recall that $G$ is said to be QSF (i.e., quasi simply filtered) if the universal cover $\tilde{X}$ of $X$ admits an exhaustion which can be "approximated" by finite simply connected CW-complexes, i.e., for every finite subcomplex $A \subset \tilde{X}$ there is a cellular map $f: Y \longrightarrow \tilde{X}$ from a finite simply connected CW-complex $Y$ which is a homeomorphism on $f^{-1}(A)$. On the other hand, $G$ is said to be WGSC (i.e., weakly geometrically simply connected) if $\tilde{X}$ has in fact an exhaustion by finite simply connected subcomplexes.

It was shown in [2] that all one-relator groups are QSF. Furthermore, the properties QSF and WGSC have recently been shown to be equivalent (see $[15,16])$. Next, in Proposition 2.7 below, we shall demonstrate a stronger property of one-relator groups (from the proper homotopy viewpoint) giving also an alternative proof of the fact that one-relator groups are semistable at infinity (see [30]). We shall need the following:

Definition 2.1. Let $X$ be a 2-dimensional (PL) CW-complex and let $d^{n}, e^{n+1}$ $\subset X$ be cells of $X, d$ being a free face of $e$ when considered as a subcomplex of $X$. An elementary internal collapse $(e, d)$ in $X$ consists of "collapsing" the cell $e$ through its face $d$, even if $d$ is not a free face of $e$ within the entire complex $X$. Of course, this implies dragging all the material adjacent to that face thus producing a new $\mathrm{CW}$-complex proper homotopy equivalent to $X$. We will refer to any of the inverses of an elementary internal collapse as an elementary internal expansion.

Definition 2.2. We say that two (possibly non-compact) 2-complexes $X$ and $Y$ are strongly proper homotopy equivalent if one is obtained from the other by a (possibly infinite) sequence of elementary internal collapses and/or expansions, in such a way that the resulting homotopy equivalence is a proper homotopy equivalence. 
Definition 2.3. Let $X$ be a 2-dimensional simply connected CW-complex. We will say that a filtration $C_{1} \subset C_{2} \subset \cdots \subset X$ of compact subcomplexes is nice if each $C_{n}$ is simply connected and, for any choice of base ray $[0, \infty) \longrightarrow X$, the fundamental pro-group

$$
\{1\} \leftarrow \pi_{1}\left(X-\operatorname{int}\left(C_{1}\right)\right) \leftarrow \pi_{1}\left(X-\operatorname{int}\left(C_{2}\right)\right) \leftarrow \cdots
$$

is a tower of finitely generated free groups of increasing bases where the bonding maps are projections.

Lemma 2.4. Let $X$ be a 2-dimensional simply connected $C W$-complex together with a nice filtration $C_{1} \subset C_{2} \subset \cdots \subset X$, and let $T_{i}, i \in I$, be a (locally finite) collection of trees inside $X$. We can get a new 2-complex $\widehat{X}$ (strongly proper homotopy equivalent to $X$ ) still containing the $T_{i}$ 's and a nice filtration $\widehat{C}_{1} \subset \widehat{C}_{2} \subset \cdots \subset \widehat{X}$ such that each intersection $\widehat{C}_{n} \cap T_{i}(n \geq 1, i \in I)$ is either empty or a connected subtree (and hence contractible).

Proof. We shall reroute certain 2-cells of $C_{i}$ up on "bridges" doubling a subforest of $\bigcup T_{j}$ in order to get connected intersections $C_{i} \cap T_{j}$. For this, consider $C_{1} \subset X$ and let $T_{i_{1}}, \ldots, T_{i_{r}} \subset X$ be those trees of the given collection which intersect $C_{1}$. We denote by $Z_{1, m} \subset T_{i_{m}}(1 \leq m \leq r)$ the smallest connected subtree containing $C_{1} \cap T_{i_{m}}$, and let $n(1) \geq 1$ be such that $Z_{1, m} \subset C_{n(1)}$, for every $1 \leq m \leq r$. Let $T_{i_{1}}, \ldots, T_{i_{r}}, T_{i_{r+1}}, \ldots, T_{i_{s}}$ be those trees of the collection which intersect $C_{n(1)}$, and take $Z_{n(1), m} \subset T_{i_{m}}$ to be either the connected subtree satisfying $Z_{1, m} \subset Z_{n(1), m} \subset C_{n(1)} \cap T_{i_{m}}$ if $1 \leq m \leq r$, or any component of $C_{n(1)} \cap T_{i_{m}}$ otherwise. We will perform in $X$ a sequence of elementary internal expansions, one for each $k$-cell $(k=0,1)$ of $\Gamma=\bigcup_{m=1}^{s}\left(C_{n(1)} \cap T_{i_{m}}-Z_{n(1), m}\right)$ as follows. First, we introduce for each 1-cell $d^{1} \subset \Gamma$ a new 2-cell $e^{2}$ having its boundary divided into two arcs, one of them corresponding to the old $d^{1}$ and the other being such that every 2-cell of $C_{n(1)}$ containing $d^{1}$ in the original complex is now being attached along this new arc. Any other 2-cell containing $d^{1}$ in the original complex is still attached along $d^{1}$ (see figure 1 ).

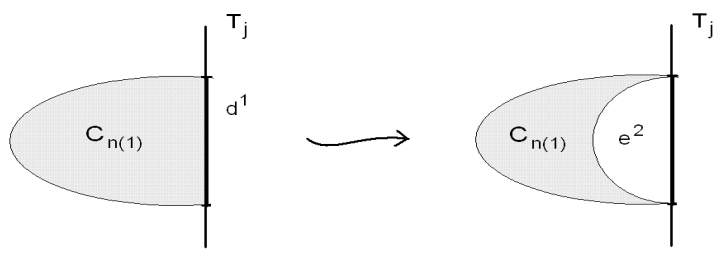

Figure 1. 
These elementary internal expansions (leaving the trees $T_{i_{m}}$ unaltered) yield a complex $X^{\prime}$ in which $C_{n(1)}$ is transformed into a new subcomplex $C_{n(1)}^{\prime} \subset X^{\prime}$ which meets each $T_{i_{m}} \subset X^{\prime}$ outside $Z_{n(1), m}$ in a finite number of vertices in $\Gamma \cap T_{i_{m}}$. Next, we introduce for each of these vertices $d^{0} \in \Gamma \cap T_{i_{m}}$ a new 1-cell $e^{1}$ with distinct boundary points, one of them corresponding to the old $d^{0}$, and such that every 2 -cell outside $C_{n(1)}^{\prime}$ containing a 1-cell of $T_{i_{m}}$ adjacent to $d^{0}$ is now also being attached along $e^{1}$, as shown in figure 2 . Thus we get a new complex $\widehat{X}^{(1)}$ (strongly proper homotopy equivalent to $X^{\prime}$ ) in which $C_{n(1)}^{\prime}$ turns into a subcomplex $\widehat{C}_{1} \subset \widehat{X}^{(1)}$ which contains $C_{1}$ and has connected intersection with each $T_{i_{m}}, 1 \leq m \leq s$. Observe that $\widehat{X}^{(1)}-\operatorname{int}\left(\widehat{C}_{1}\right)$ is strongly proper homotopy equivalent to $X-\operatorname{int}\left(C_{n(1)}\right)$, by construction.

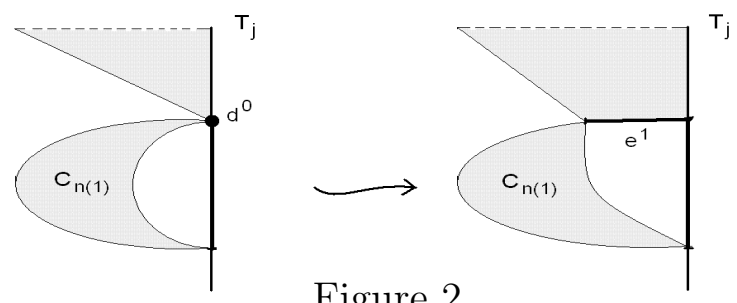

Figure 2.

Let $C_{1}^{(1)} \subset C_{2}^{(1)} \subset \cdots \subset \widehat{X}^{(1)}$ be the obvious filtration obtained from $C_{1} \subset C_{2} \subset \cdots \subset X$ (i.e., each $C_{n}$ "expands" to $C_{n}^{(1)}$ ), and take $N \geq 1$ such that $\widehat{C}_{1} \subset C_{N}^{(1)}$. We apply to $C_{N}^{(1)} \subset \widehat{X}^{(1)}$ the same argument we applied to $C_{1} \subset X$ so as to get a new complex $\widehat{X}^{(2)}$ (via a strong proper homotopy equivalence with $\widehat{X}^{(1)}$ which leaves fixed a compact subcomplex containing $C_{N}^{(1)} \supset \widehat{C}_{1}$ ) and a subcomplex $\widehat{C}_{2} \supset C_{N}^{(1)} \supset \widehat{C}_{1}$ with the required properties. Iterating this process we obtain the desired 2-complex $\widehat{X}$ (as the limit of the complexes $\left.\widehat{X}^{(i)}, i \geq 1\right)$ and a nice filtration $\widehat{C}_{1} \subset \widehat{C}_{2} \subset \cdots \subset \widehat{X}$.

Remark 2.5. Note that the strong homotopy equivalence $X \longrightarrow \widehat{X}$ obtained in Lemma 2.4 maps every tree in $X$ to another tree in $\widehat{X}$, by construction.

Next we introduce some notation. Let $G$ be a finitely generated onerelator group and $P=\langle X ; R\rangle$ be any (finite) presentation of $G$ with a single defining relation $R=Q^{s}$ ( $s$ maximal) which is assumed to be a cyclically reduced word. We denote by $K_{P}$ the standard (compact) 2-dimensional $\mathrm{CW}$-complex associated to this presentation. Note that $K_{P}^{1}$ is a bouquet of circles consisting of a 1-cell $e_{i}$ for each element of the basis $x_{i} \in X$, all of them sharing the single vertex in $K_{P}$. Finally, $K_{P}$ is obtained from $K_{P}^{1}$ by attaching a 2-cell $d$ via a PL map $S^{1} \longrightarrow K_{P}^{1}$ which is the composition of the map $z \in S^{1} \mapsto z^{s} \in S^{1}$ and a PL map $f_{Q}: S^{1} \longrightarrow K_{P}^{1}$ which spells out the word $Q$. 
Remark 2.6. Note that every lift in the universal cover $\tilde{d} \subset \tilde{K}_{P}$ of the 2-cell $d \subset K_{P}$ is a disk (as $R$ is cyclically reduced). Moreover, by the Magnus' Freiheitssatz (see $[25,27]$ ) every subcomplex of the 1-skeleton $K_{P}^{1}$ not containing all the 1-cells involved in the relator $R$ lifts in the universal cover $\tilde{K}_{P}$ to a disjoint union of trees.

Proposition 2.7. Given any finite one-relator group presentation $P$ as above, the universal cover $\tilde{K}_{P}$ of the standard 2-complex $K_{P}$ is strongly proper homotopy equivalent to another 2-complex $\widehat{K}_{P}$ which admits a nice filtration $\widehat{C}_{1} \subset \widehat{C}_{2} \subset \cdots \subset \widehat{K}_{P}$.

Proof. The proof is modelled after ([12], Thm. 2.1), and the method we use goes back to Magnus [26]. With the notation above, we set $n=$ length $(Q)$. Note that if $n=1$ then $R=x_{i_{0}}{ }^{ \pm s}$ for some $x_{i_{0}} \in X$, and $\tilde{K}_{P}$ is the universal cover of the bouquet $K_{\mathbb{Z}_{s}} \vee\left(\vee_{i \neq i_{0}} e_{i}\right)$, where $K_{\mathbb{Z}_{s}}$ is the standard 2-complex associated to the obvious presentation of $\mathbb{Z}_{s}$. The 2-complex $\widehat{K}_{P}=\tilde{K}_{P}$ clearly satisfies the required properties, and its fundamental progroup corresponds to the trivial tower. As an example, the universal cover $\tilde{K}_{P}$ is depicted in figure 3 for the presentation $P=\left\langle a, b ; a^{2}\right\rangle$ of $\mathbb{Z} * \mathbb{Z}_{2}$, indicating in dark color the first two subcomplexes of a (nice) filtration $\widehat{C}_{1} \subset \widehat{C}_{2} \subset \cdots \subset \tilde{K}_{P}$.
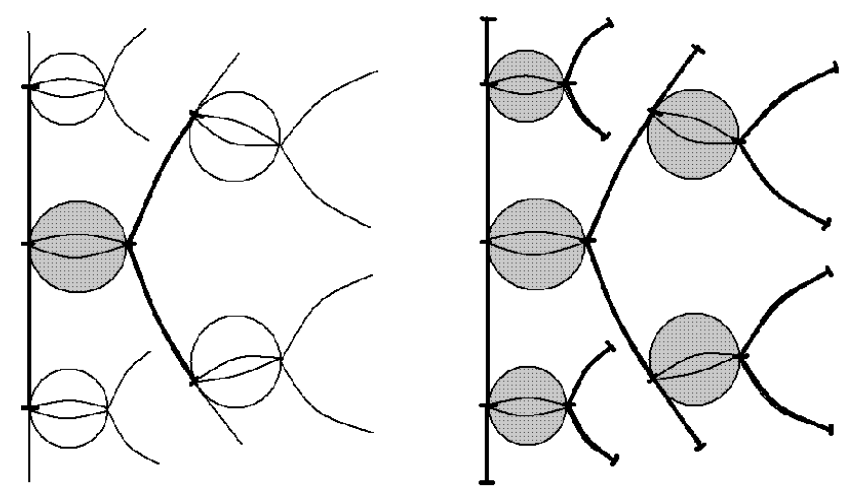

Figure 3.

Assume inductively the following two statements hold for any (finite) group presentation $P^{\prime}=\left\langle X^{\prime} ; R^{\prime}\right\rangle$ with a single defining relation $R^{\prime}=Q^{\prime s}$ (s maximal) which is a cyclically reduced word with $k=\operatorname{length}\left(Q^{\prime}\right) \leq n-1$ :

(Case 1$)_{k}$ : If there is $x_{i_{0}} \in X^{\prime}$ which occurs in $Q^{\prime}$ with exponent sum $\sigma_{Q^{\prime}}\left(x_{i_{0}}\right)=0$ then the conclusion of Proposition 2.7 follows for $\tilde{K}_{P^{\prime}}$.

(Case 2$)_{k}$ : If the word $Q^{\prime}$ contains no generators with exponent sum zero then the conclusion of Proposition 2.7 follows for $\tilde{K}_{P^{\prime}}$. 
Furthermore, we assume in addition that for any such $P^{\prime}$ the following condition is satisfied:

${ }^{*}$ ) Every tree in the 1 -skeleton of $\tilde{K}_{P^{\prime}}$ gets mapped (under the strong proper homotopy equivalence) to another tree in the final 2-complex $\widehat{K}_{P^{\prime}}$.

Next we proceed to show case $k=n$ by proving that the statements $(\text { Case } 1)_{n}$ and (Case 2$)_{n}$ are true as well as condition $\left(^{*}\right)$ in each of them. Due to the rather long proofs of both cases we deal with them separately in the two subsequent sections.

Remark 2.8. It is well-known that if the one-relator $G$ is torsion-free then the universal cover $\tilde{K}_{P}$ of $K_{P}$ is a contractible 2-dimensional $C W$-complex, by a result of Dyer and Vasquez [12] which can be thought of as a geometric version of Lyndon's Identity Theorem [24] (see also [10]). Therefore, $\widehat{K}_{P}$ is also contractible and $\widehat{C}_{1} \subset \widehat{C}_{2} \cdots \subset \widehat{K}_{P}$ is a nice filtration consisting of compact simply connected acyclic, and hence contractible, subcomplexes.

In case $G$ has torsion we still find a contractible subcomplex $\widehat{L}_{P} \subset \widehat{K}_{P}$ carrying the fundamental pro-group (i.e., pro $-\pi_{1}\left(\widehat{L}_{P}\right) \cong$ pro $-\pi_{1}\left(\widehat{K}_{P}\right)$ ) and a nice filtration $\widehat{L}_{1} \subset \widehat{L}_{2} \subset \cdots \subset \widehat{L}_{P}$ consisting of compact contractible subcomplexes. Indeed, fix a lift $\tilde{d} \subset \tilde{K}_{P}$ of the 2-cell $d \subset K_{P}$, and observe that $\tilde{d}$ and all its translates $\tilde{d} \cdot g \subset \tilde{K}_{P}$ (by the $G$-action) are copies of the disk $D^{2}$ (as $R$ is cyclically reduced), which come together in subcomplexes $L_{k}=\tilde{d} \cdot g_{k} \cup \tilde{d} \cdot\left(g_{k} Q\right) \cup \cdots \cup \tilde{d} \cdot\left(g_{k} Q^{s-1}\right) \subset \tilde{K}_{P}, g_{k} \in G$, consisting of $s$ disks attached along their boundaries via the identity map. It is worth noting that the only non-trivial 2-cycles in $\tilde{K}_{P}$ are those which are (finite) combinations of non-trivial 2-cycles in some of the $L_{k}$ 's. This is due to the fact that the relation module (associated with the given presentation of $G$ ) is a cyclic $\mathbb{Z} G$-module with one obvious relation (see $[9,3]$ ). Next, we consider a subcomplex $L_{P} \subset \tilde{K}_{P}$ containing only one 2-cell from each of the subcomplexes $L_{k} \subset \tilde{K}_{P}$ described above. Note that then $L_{P}$ is contractible, and one can check that the proof of Proposition 2.7 produces a new 2complex $\widehat{L}_{P} \subset \widehat{K}_{P}$ (strongly proper homotopy equivalent to $L_{P}$ ) together with a nice filtration $\widehat{C}_{1} \cap \widehat{L}_{P} \subset \widehat{C}_{2} \cap \widehat{L}_{P} \subset \cdots \subset \widehat{L}_{P}$ consisting of compact contractible subcomplexes.

\section{Proof that $(\text { Case } 1)_{\leq n-1}+(\text { Case } 2)_{\leq n-1} \Rightarrow(\text { Case } 1)_{n}$}

The purpose of this section is to prove $(\text { Case } 1)_{n}$. In fact, we shall prove the conclusion is true for any (finite) one-relator group presentation $P=\langle X ; R\rangle$, $R=Q^{s}$ (s maximal), assuming $R$ is cyclically reduced and there is $x_{i_{0}} \in X$ which occurs in $Q$ with exponent sum $\sigma_{Q}\left(x_{i_{0}}\right)=0$ such that the word obtained from $Q$ by deleting the symbols $x_{i_{0}}$ and $x_{i_{0}}^{-1}$ has length $\leq n-1$. 
We shall divide the proof into the following four steps (we keep the notation for the cells of $K_{P}$ from $\S 2$ ).

1. Notation and some preliminaries. For each integer $k$, let $J_{k}$ be a copy of the bouquet $\vee_{i \neq i_{0}} e_{i} \subset K_{P}$ (with the 0 -cell of $K_{P}$ as base point) and take $L$ to be the space obtained by attaching the space $J_{k}$ to the real line $\mathbb{R}$ at each integer point $k$ (through its base point). Let $h: L \longrightarrow L$ be the PL homeomorphism which takes a point of $J_{k}$ to the corresponding point of $J_{k+1}$ and sends $x \in \mathbb{R}$ to $x+1 \in \mathbb{R}$, and denote by $p: L \longrightarrow L / H \equiv K_{P}^{1}$ the resulting covering map, where $H$ is the infinite cyclic subgroup of selfhomeomorphisms generated by $h$. Moreover, one can check that there is a pointed PL map $f^{\prime}: S^{1} \longrightarrow L$ (taking $0 \in \mathbb{R}$ as the base point of $L$ ) such that $p f^{\prime}=f_{Q}$ (=the given PL map $S^{1} \longrightarrow K_{P}^{1}$ which spells out the word $Q$ ) and $f^{\prime}$ spells out a cyclically reduced word $Q^{\prime}$ (with length $\left(Q^{\prime}\right)<n$ ) in the free group $F(Y)$, where $Y$ is the set of generators for $\pi_{1}(L,\{0\})$ obtained from those for each space $J_{k}$ via the obvious base point changes (see [12]). Let $u, v \in \mathbb{Z}$ be the integers such that $[u, v] \subset \mathbb{R}$ is the smallest interval satisfying $f^{\prime}\left(S^{1}\right) \subset \mathbb{R} \cup \bigcup_{k \in[u, v]} J_{k}$. Let $K_{P}^{\prime}$ be the space obtained from $L$ by gluing disks $D_{k}^{2}$ via the composition $f_{k}^{\prime \prime}$ of the map $z \mapsto z^{s}$ with the PL map $f_{k}^{\prime}=h^{k} f^{\prime}: S^{1} \longrightarrow L, k \in \mathbb{Z}$. We keep denoting by $H$ the infinite cyclic subgroup of self-homeomorphisms of $K_{P}^{\prime}$ generated by the obvious extension of $h$. Thus, we have an (intermediate) covering space $p: K_{P}^{\prime} \longrightarrow K_{P}^{\prime} / H \equiv$ $K_{P}$ (corresponding to the kernel of the homomorphism $F(X) / N(R) \longrightarrow \mathbb{Z}$ induced by $\left.F(X) \longrightarrow \mathbb{Z}, w \mapsto \sigma_{w}\left(x_{i_{0}}\right)\right)$, and we denote by $q: \tilde{K}_{P} \longrightarrow K_{P}^{\prime}$ the corresponding universal covering map. Let $K_{m}^{\prime} \subset K_{P}^{\prime}$ be the subcomplex consisting of the real line $\mathbb{R}$ together with $D_{m}^{2} \cup_{f_{m}^{\prime \prime}}\left(\bigcup_{k \in[u+m, v+m]} J_{k}\right)$. Note that, as the interval $[u+m, v+m]$ can be shrunk to a point, $K_{m}^{\prime}$ is homotopy equivalent to the wedge $K_{P^{\prime}} \vee \mathbb{R}$ where $K_{P^{\prime}}$ is the standard 2-dimensional CW-complex associated to a group presentation $P^{\prime}=\left\langle X^{\prime} ; Q^{\prime s}\right\rangle$ where $Q^{\prime}$ is as above and $X^{\prime} \subset Y$ is the subset consisting of those generators contained in $K_{m}^{\prime}$.

2. The structure of the universal cover $\tilde{K}_{P}$. It follows from ([12], Sublemma 3.2.2) that the inclusion $K_{m}^{\prime} \subset K_{P}^{\prime}(m \in \mathbb{Z})$ induces an injection of fundamental groups (this need not hold if $R=Q^{s}$ has not been cyclically reduced, see Remark 3.2). In this way, each component of the preimage $q^{-1}\left(K_{m}^{\prime}\right) \subset \widetilde{K}_{P}$ is a copy of the universal cover of $K_{m}^{\prime}$. On the other hand, as $K_{P}^{\prime}=\bigcup_{m \in \mathbb{Z}} K_{m}^{\prime}$, it is not hard to see that the universal cover $\widetilde{K}_{P}$ then consists of collections of copies of the universal covers of the spaces $K_{m}^{\prime}$ $(m \in \mathbb{Z})$, appropriately glued together along a certain collection of trees. More precisely, observe that for every $m>n, K_{m}^{\prime} \cap K_{n}^{\prime}=\mathbb{R}$ if $m-n>v-u$; otherwise, $K_{m}^{\prime} \cap K_{n}^{\prime} \subset \mathbb{R} \cup J_{u+m} \cup \cdots \cup J_{v+n}$ is a graph with the property 
that the inclusion $K_{m}^{\prime} \cap K_{n}^{\prime} \subset K_{m}^{\prime}$ (or $K_{n}^{\prime}$ ) induces an injection of the fundamental groups, by the choice of the integers $u, v$. Thus, each component of the preimage $q^{-1}\left(K_{m}^{\prime} \cap K_{n}^{\prime}\right) \subset \widetilde{K}_{P}$ is a copy of the universal cover of $K_{m}^{\prime} \cap K_{n}^{\prime}$ which is a tree by an application of the Magnus' Freiheitssatz to the presentation $P^{\prime}=\left\langle X^{\prime} ; Q^{\prime s}\right\rangle$, as the generators of $X^{\prime}$ which occur in $K_{m}^{\prime} \cap K_{n}^{\prime}$ are not all of those involved in the relator (see Remark 2.6). Moreover, if more than two copies of the universal covers of some of the subcomplexes $K_{m}^{\prime}$ have non-empty intersection in $\widetilde{K}_{P}$ then this intersection must be a subtree of the intersection of any two of them.

3. Altering $\widetilde{K}_{P}$ within its (strong) proper homotopy type. Next we check that the universal cover of each complex $K_{m}^{\prime}$ is strongly proper homotopy equivalent to another 2-complex $\widehat{K}_{m}^{\prime}$ which admits a nice filtration. To this end, observe that the universal cover $\widetilde{K}_{P^{\prime}}$ of $K_{P^{\prime}}\left(P^{\prime}\right.$ as in Step 1) has this property by the inductive hypothesis. Moreover, the universal cover of $K_{m}^{\prime}$ is obtained from the universal cover of $K_{P^{\prime}} \vee \mathbb{R}$ by "expanding" in an appropriate way each vertex to an interval $[u+m, v+m]$ so as to recover the subgraph $K_{m}^{\prime} \cap L$.

We perform in $\widetilde{K}_{P}$ all the elementary internal collapses and/or expansions needed for passing from each copy of the universal cover of $K_{m}^{\prime}(m \in \mathbb{Z})$ inside $\widetilde{K}_{P}$ to the corresponding 2-complex $\widehat{K}_{m}^{\prime}$. In this way we obtain a new 2-complex $\widehat{K}_{P}^{\prime}$ (strongly proper homotopy equivalent to $\widetilde{K}_{P}$ and still containing a copy of $q^{-1}(\mathbb{R})$ ) which consists of collections of copies of the complexes $\widehat{K}_{m}^{\prime}(m \in \mathbb{Z})$ glued together appropriately along the corresponding collection of trees obtained from those in the construction of $\widetilde{K}_{P}$, according to condition $\left(^{*}\right)$ of the inductive hypothesis (see $\S 2$ ). For every $m \in \mathbb{Z}$ and every copy of $\widehat{K}_{m}^{\prime}$ inside $\widehat{K}_{P}^{\prime}$ we consider a nice filtration $C_{1, m} \subset C_{2, m} \subset \cdots \subset \widehat{K}_{m}^{\prime}$ and denote by $T_{1}, T_{2}, \ldots, T_{s}$ those trees along which the given copy of $\widehat{K}_{m}^{\prime}$ intersects with a copy (inside $\left.\widehat{K}_{P}^{\prime}\right)$ of any other subcomplex $\widehat{K}_{n}^{\prime}(n \neq m)$. Let $\widehat{C}_{1, m} \subset \widehat{C}_{2, m} \subset \cdots \subset \widehat{K}_{m}$ be the 2-complex and the nice filtration obtained from $C_{1, m} \subset C_{2, m} \subset \cdots \subset \widehat{K}_{m}^{\prime}$ and the collection of trees $T_{1}, T_{2}, \ldots, T_{s}$ proceeding as in Lemma 2.4. In particular, each intersection $\widehat{C}_{n, m} \cap T_{i}$ $(n \geq 1,1 \leq i \leq s)$ is either empty or connected (and hence contractible).

Let $\widehat{K}_{P}$ be the 2-complex (strongly proper homotopy equivalent to $\widehat{K}_{P}^{\prime}$ and hence to $\widetilde{K}_{P}$ ) obtained from collections of copies of the complexes $\widehat{K}_{m}$ $(m \in \mathbb{Z})$ glued together as follows : a copy of $\widehat{K}_{m}$ is being glued to a copy of $\widehat{K}_{n}$ along a tree $T$ whenever the corresponding copies of $\widehat{K}_{m}^{\prime}$ and $\widehat{K}_{n}^{\prime}$ inside $\widehat{K}_{P}^{\prime}$ are glued together along the corresponding copy of the same tree $T$.

Note that in the process of altering $\widetilde{K}_{p}$ to $\widehat{K}_{P}$ we have used inductive hypothesis and applications of Lemma 2.4. This way we ensure that condition $\left(^{*}\right)$ gets satisfied (see Remark 2.5). 
4. Building the required filtration for $\widehat{K}_{P}$. We must now build a nice filtration for $\widehat{K}_{P}$, and this will finish the proof. We keep the notation from Step 3. Fix $m \in Z$ and a copy of $\widehat{K}_{m}$ in $\widehat{K}_{P}$, and consider $\widehat{C}_{1, m} \subset \widehat{K}_{m}$. By abuse of notation, we will denote by $\widehat{K}_{m(1)}, \ldots, \widehat{K}_{m\left(p_{1}\right)}$ those different copies in $\widehat{K}_{P}$ of the corresponding complexes which intersect the chosen copy of $\widehat{K}_{m}$ at points of $\widehat{C}_{1, m}$ (i.e., if $m(i)=m(j)$ for some $1 \leq i<j \leq p_{1}$, the complexes $\widehat{K}_{m(i)}, \widehat{K}_{m(j)}$ above are considered different copies of the same complex). Take $N_{1} \geq 1$ such that $\widehat{C}_{N_{1}, m(j)} \cap \widehat{K}_{m} \supset \widehat{C}_{1, m} \cap \widehat{K}_{m(j)}$ (both intersections being connected subtrees by hypothesis), $1 \leq j \leq p_{1}$. Set $\widehat{C}_{1}=\widehat{C}_{1, m} \cup\left(\bigcup_{j=1}^{p_{1}} \widehat{C}_{N_{1}, m(j)}\right) \subset \widehat{K}_{P}$ which is easily shown to be simply connected, as the simply connected compact subcomplexes $\widehat{C}_{\alpha, \beta}$ intersect each other along connected subtrees of those trees used in the construction of $\widehat{K}_{P}$ (see Step 3). Moreover, by the generalized Van Kampen's argument, as $\widehat{C}_{1, m}$ and each $\widehat{C}_{N_{1}, m(j)}$ are members of a nice filtration of the corresponding copy of $\widehat{K}_{m}$ and $\widehat{K}_{m(j)}$ respectively and (every copy of) any other subcomplex $\widehat{K}_{\lambda} \subset \widehat{K}_{P}$ is simply connected, one can check that (choosing base points on any given base ray in $\left.\widehat{K}_{P}\right)$ the fundamental group of $\widehat{K}_{P}-\operatorname{int}\left(\widehat{C}_{1}\right)$ is indeed a free product of the fundamental groups of $\widehat{K}_{P}-\operatorname{int}\left(\widehat{C}_{1, m}\right)$ and $\widehat{K}_{P}-\operatorname{int}\left(\widehat{C}_{N_{1}, m(j)}\right)$ together with an extra free group (of finite rank) coming from the intersection of $\widehat{C}_{1, m}$ and each $\widehat{C}_{N_{1}, m(j)}$ with any other subcomplex $\widehat{K}_{\lambda} \subset \widehat{K}_{P}$.

Consider now $\widehat{C}_{2, m} \subset \widehat{K}_{m}$. We denote by $\widehat{K}_{m(1)}, \ldots, \widehat{K}_{m\left(p_{2}\right)}\left(p_{2} \geq p_{1}\right)$ those different copies in $\widehat{K}_{P}$ of the corresponding complexes which intersect the given copy of $\widehat{K}_{m}$ at points of $\widehat{C}_{2, m}$, and take $N_{2} \geq N_{1}$ such that $\widehat{C}_{N_{2}, m(j)} \cap$ $\widehat{K}_{m} \supset \widehat{C}_{2, m} \cap \widehat{K}_{m(j)}$ (both being connected subtrees), $1 \leq j \leq p_{2}$. Next, for each $1 \leq j \leq p_{2}$, we denote by $\widehat{K}_{m(j, 1)}, \ldots, \widehat{K}_{m\left(j, q_{j}\right)}$ those different copies in $\widehat{K}_{P}$ of the corresponding complexes which intersect the given copy of $\widehat{K}_{m(j)}$ at points of $\widehat{C}_{N_{2}, m(j)}$, and take $N_{3} \geq N_{2}$ such that $\widehat{C}_{N_{3}, m(j, l)} \cap \widehat{K}_{m(j)} \supset$ $\widehat{C}_{N_{2}, m(j)} \cap \widehat{K}_{m(j, l)}$ (both being connected subtrees), $1 \leq l \leq q_{j}$. Set $\widehat{C}_{2}=$ $\widehat{C}_{2, m} \cup\left(\bigcup_{j=1}^{p_{2}} \widehat{C}_{N_{2}, m(j)}\right) \cup\left(\bigcup_{j=1}^{p_{2}} \bigcup_{l=1}^{q_{j}} \widehat{C}_{N_{3}, m(j, l)}\right) \subset \widehat{K}_{P}$ which is again simply connected and contains $\widehat{C}_{1}$, by construction.

As before, one can also check that the fundamental group of $\widehat{K}_{P}-i n t\left(\widehat{C}_{2}\right)$ is finitely generated and free. Finally, repeating this process (starting off with the successive $\widehat{C}_{n, m} \subset \widehat{K}_{m}$ and going each time one step further inside $\left.\widehat{K}_{P}\right)$ we get a filtration $\widehat{C}_{1} \subset \widehat{C}_{2} \subset \cdots \subset \widehat{K}_{P}$ of compact simply connected subcomplexes. Moreover, choosing base points on any given base ray, one can easily check that $\operatorname{rank}\left(\pi_{1}\left(\widehat{K}_{P}-\operatorname{int}\left(\widehat{C}_{n+1}\right)\right)\right) \geq \operatorname{rank}\left(\pi_{1}\left(\widehat{K}_{P}-\operatorname{int}\left(\widehat{C}_{n}\right)\right)\right)$ 
and the homomorphism $\pi_{1}\left(\widehat{K}_{P}-\operatorname{int}\left(\widehat{C}_{n+1}\right)\right) \longrightarrow \pi_{1}\left(\widehat{K}_{P}-\operatorname{int}\left(\widehat{C}_{n}\right)\right)$ can be taken to be a projection between finitely generated free groups.

Remark 3.1. Notice that the argument used for the (inductive) proof of (Case 1$)_{n}$ shows that the inverse image $(q \circ p)^{-1}\left(e_{i_{0}}\right) \subset \widetilde{K}_{P}$ of the 1-cell $e_{i_{0}} \subset K_{P}$ in Step 1 remains as a subcomplex of the final 2-complex $\widehat{K}_{P}$.

Remark 3.2. Consider the finite group presentation $P=\left\langle a, b ; a^{-1} b a^{-1} b^{-1} a\right\rangle$ with a single defining relation $R=a^{-1} b a^{-1} b^{-1} a(R=Q, s=1)$ which is not a cyclically reduced word in the free group $F(\{a, b\})$. Let $K_{P}$ be the standard 2-complex associated with this group presentation, and let $K_{P}^{\prime}$ and $K_{m}^{\prime}(m \in \mathbb{Z})$ be as above (here, $\left.x_{i_{0}}=b\right)$. It is easy to see that in this case $K_{P}^{\prime}=\widetilde{K}_{P}$ and the inclusion $K_{m}^{\prime} \subset K_{P}^{\prime}$ does not induce an injection between the fundamental groups (see figure 4 ). On the other hand, this example shows a compact 2-dimensional CW-complex $K_{P}$ with $\pi_{1}\left(K_{P}\right) \cong \mathbb{Z}$ whose universal cover can not be written as an increasing union of compact simply connected subcomplexes.

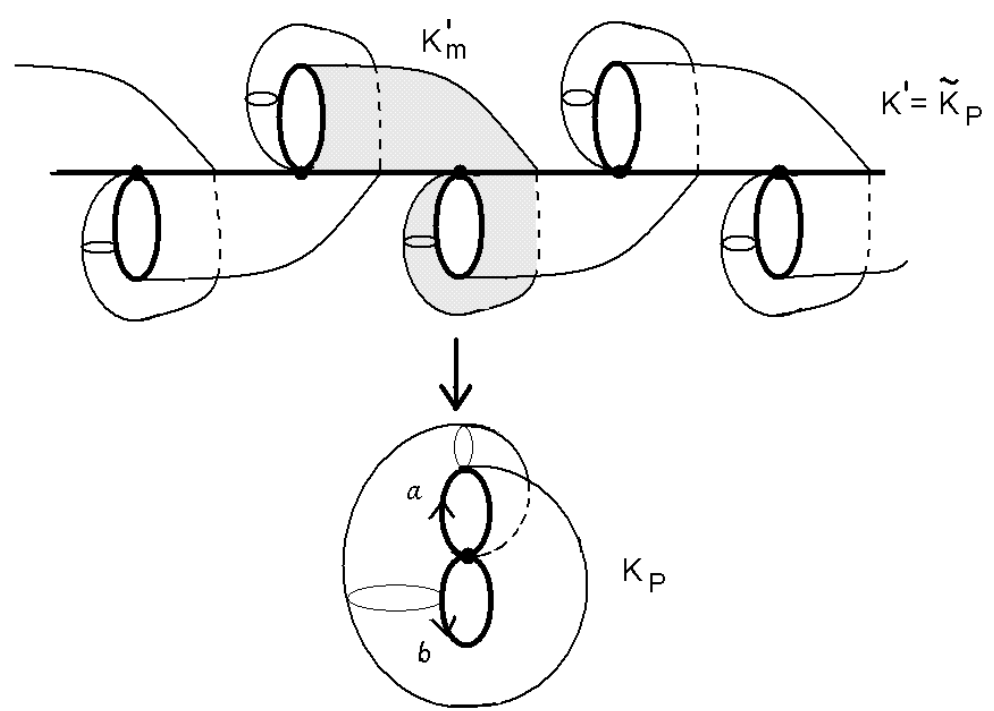

Figure 4.

\section{Proof that $(\text { Case } 1)_{\leq n-1}+(\text { Case } 2)_{\leq n-1} \Rightarrow(\text { Case } 2)_{n}$}

The purpose of this section is to prove $(\text { Case } 2)_{n}$ for any (finite) one-relator group presentation $P=\langle X ; R\rangle$ where $R=Q^{s}$ ( $s$ maximal) is assumed to be a cyclically reduced word with length $(Q)=n \geq 2$. Thus, suppose $Q$ contains no generators with exponent sum 0 . We divide the proof into the following three steps. 
1. Notation. Since length $(Q)=n \geq 2$ it follows that $Q$ must involve at least two generators $x_{i_{0}}, x_{i_{1}} \in X$. We introduce new symbols $A, B \notin F(X)$ and take $X^{\prime}=\left(X-\left\{x_{i_{0}}\right\}\right) \cup\{A\}$ and $X^{\prime \prime}=\left(X-\left\{x_{i_{0}}, x_{i_{1}}\right\}\right) \cup\{A, B\}$. Consider the group presentations $P^{\prime}=\left\langle X^{\prime} ; Q^{\prime s}\right\rangle$ and $P^{\prime \prime}=\left\langle X^{\prime \prime} ; Q^{\prime \prime s}\right\rangle$, where $Q^{\prime}$ is obtained from $Q$ by replacing $x_{i_{0}}$ with $A^{q}\left(q=\sigma_{Q}\left(x_{i_{1}}\right)\right.$, the exponent sum of $x_{i_{1}}$ in $Q$ ), and $Q^{\prime \prime}$ is obtained from $Q^{\prime}$ by replacing $x_{i_{1}}$ with $B A^{-p}$ $\left(p=\sigma_{Q}\left(x_{i_{0}}\right)\right)$. The words $Q^{\prime}, Q^{\prime \prime}$ are not proper powers and $\sigma_{Q^{\prime \prime}}(A)=0$. Furthermore, we may assume $Q^{\prime \prime}$ cyclically reduced, since the associated 2complex $K_{P^{\prime \prime}}$ will only change up to homotopy and its universal cover will only change up to strong proper homotopy.

2. The universal covers $\widetilde{K}_{P^{\prime}}, \widetilde{K}_{P^{\prime \prime}}$ and their relation with $\widetilde{K}_{P}$. Observe that the word obtained from $Q^{\prime \prime}$ by deleting the symbols $A$ and $A^{-1}$ has length less than $n=$ length $(Q)$, and hence the induction hypothesis together with the argument used in $\S 3$ yields that the universal cover of $K_{P^{\prime \prime}}$ is strongly proper homotopy equivalent to a 2-complex $\widehat{K}_{P^{\prime \prime}}$ which admits a nice filtration. According to [12], $K_{P^{\prime}}$ and $K_{P^{\prime \prime}}$ are homotopy equivalent, and hence their universal covers $\widetilde{K}_{P^{\prime}}$ and $\widetilde{K}_{P^{\prime \prime}}$ are proper homotopy equivalent. In fact, one can easily describe this proper homotopy equivalence geometrically as follows (see figure 5 ). The complex $\widetilde{K}_{P^{\prime}}$ is obtained from $\widetilde{K}_{P^{\prime \prime}}$ by sliding the final endpoint $\beta(1)$ of each lift $\beta \subset \widetilde{K}_{P^{\prime \prime}}$ of the (oriented) generating circle corresponding to $B$ (dragging the material of the 2-cells involved, thus substituting the old one) over the edge path $\alpha, \alpha \cdot A^{-1}, \ldots, \alpha \cdot A^{-p+1} \subset \widetilde{K}_{P^{\prime \prime}}$, where $\alpha$ is the lift of the inverse path of the (oriented) generating circle corresponding to $A$ whose initial endpoint $\alpha(0)$ coincides with $\beta(1)$. Note that as $A$ generates an infinite cyclic subgroup in both group presentations $P^{\prime}$ and $P^{\prime \prime}$, the support of each of the above edge paths is homeomorphic to a closed interval.
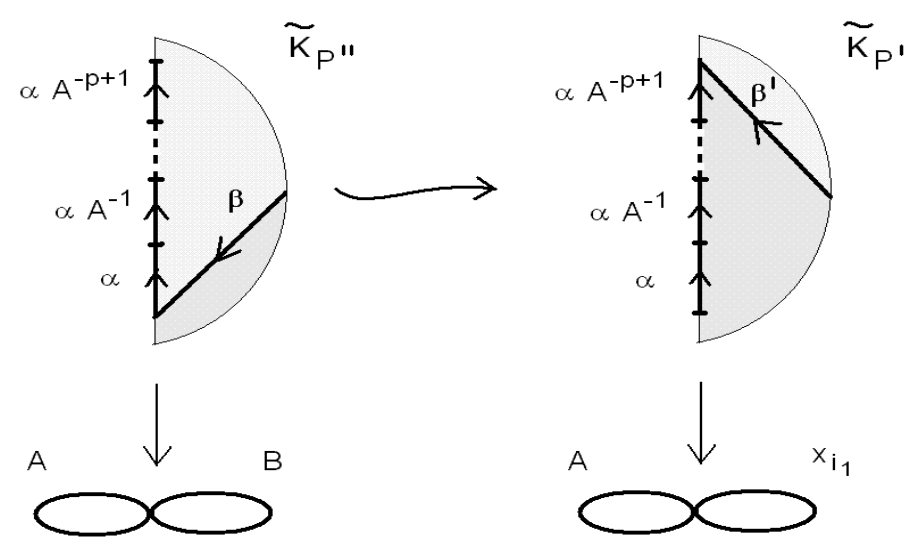

Figure 5. 
It is not hard to see from this description that $\widetilde{K}_{P^{\prime}}$ and $\widetilde{K}_{P^{\prime \prime}}$ are in fact strongly proper homotopy equivalent. It remains to show that $\widetilde{K}_{P}$ indeed has the required property.

Let $e_{i_{0}} \subset K_{P}$ be the 1-cell corresponding to the generator $x_{i_{0}}$, and let $M$ be the mapping cylinder of a map $e_{i_{0}} \longrightarrow S^{1}$ of degree $q$. Then, the adjunction complex $W=K_{P} \cup_{e_{i_{0}}} M$ is homotopy equivalent to $K_{P^{\prime}}$ and hence their universal covers $\widetilde{W}$ and $\widetilde{K}_{P^{\prime}}$ are proper homotopy equivalent. In fact, $\widetilde{W}$ is built from copies of $\widetilde{K}_{P}$ and $Y_{q} \times \mathbb{R}$ glued together appropriately along $\operatorname{Fr}\left(Y_{q} \times \mathbb{R}\right)$, where $Y_{q} \subset \mathbb{R}^{2}$ consists of $q$ segments $\left[u, v_{i}\right], 1 \leq i \leq q$, sharing a common vertex $u$. From here, one gets the universal cover $\widetilde{K}_{P^{\prime}}$ by shrinking each copy of $Y_{q} \times \mathbb{R}$ to its centerline $\{u\} \times \mathbb{R}$ (projecting down onto the base of $M$ ) which gets identified with each connected component of $\left(q^{\prime}\right)^{-1}(A) \subset \widetilde{K}_{P^{\prime}}$, where $q^{\prime}: \widetilde{K}_{P^{\prime}} \longrightarrow K_{P^{\prime}}$ is the universal covering map and $A \subset K_{P^{\prime}}$ is the circle (replacing $e_{i_{n}} \subset K_{P}$ ) representing the basis element $A \in X^{\prime}(\mathrm{s}$

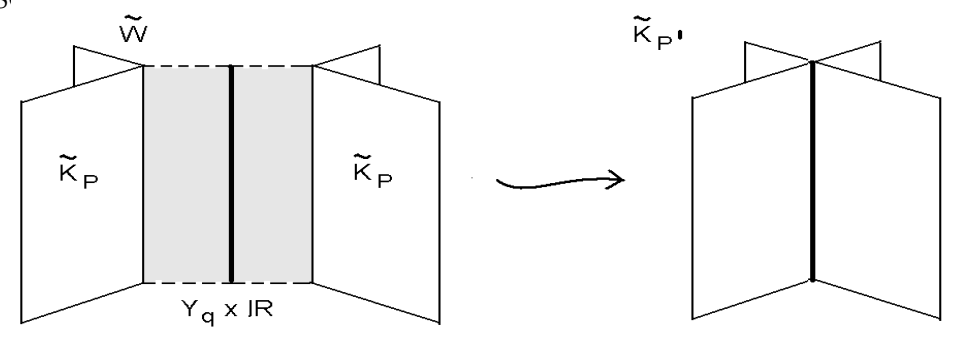

Figure 6.

Notice that each component of $\left(q^{\prime}\right)^{-1}(A)$ is a line and the subcomplex $\left(q^{\prime}\right)^{-1}(A) \subset \widetilde{K}_{P^{\prime}}$ remains unaltered when passing from $\widetilde{K}_{P^{\prime}}$ to $\widetilde{K}_{P^{\prime \prime}}$ and from $\widetilde{K}_{P^{\prime \prime}}$ to $\widehat{K}_{P^{\prime \prime}}$ (see Remark 3.1 ). It is easy to see that $\widetilde{W}$ is strongly proper homotopy equivalent to $\widetilde{K}_{P^{\prime}}$.

3. Altering $\widetilde{K}_{P}$ to $\widehat{K}_{P}$ and getting a nice filtration. Choose a copy of $\widetilde{K}_{P}$ in $\widetilde{W}$ and denote by $\widehat{K}_{P} \subset \widehat{K}_{P^{\prime \prime}}$ the 2-complex that $\widetilde{K}_{P}$ ends up being strongly proper homotopy equivalent to when performing all the elementary internal collapses and/or expansions in order to get $\widetilde{W} \sim \widetilde{K}_{P^{\prime}} \sim \widetilde{K}_{P^{\prime \prime}} \sim$ $\widehat{K}_{P^{\prime \prime}}$ (here, " $\sim$ "stands for strong proper homotopy equivalence). Observe that the composition $\widetilde{W} \longrightarrow \widehat{K}_{P \text { " }}$ of these strong homootopy equivalences maps every tree in $\widetilde{W}$ to another tree in $\widehat{K}_{P^{\prime \prime}}$ (by construction), and hence condition $(*)$ gets satisfied (see $\S 2$ ). Finally, given a nice filtration $\widehat{C}_{1}^{\prime \prime} \subset$ $\widehat{C}_{2}^{\prime \prime} \subset \cdots \subset \widehat{K}_{P^{\prime \prime}}$, we may assume that the intersection of each $\widehat{C}_{i}^{\prime \prime}$ with every component of $\left(q^{\prime}\right)^{-1}(A)$ is either empty or connected, by an application of Lemma 2.4 (and replacing $\widehat{K}_{P^{\prime \prime}}$ if necessary). Given the above, one can get a nice filtration $\widehat{C}_{1} \subset \widehat{C}_{2} \subset \cdots \subset \widehat{K}_{P}$ where each $\widehat{C}_{i}$ can be chosen to be the 
intersection $\widehat{C}_{i}^{\prime \prime} \cap \widehat{K}_{P}, i \geq 1$. Indeed, one can check that the fundamental group of each component $\widehat{J}$ of $\widehat{K}_{P}-\operatorname{int}\left(\widehat{C}_{i}\right)$ is a free factor of the fundamental group of the corresponding component $\widehat{J}^{\prime \prime} \supset \widehat{J}$ of $\widehat{K}_{P^{\prime \prime}}-\operatorname{int}\left(\widehat{C}_{i}^{\prime \prime}\right)\left(\operatorname{ascl}\left(\widehat{J}^{\prime \prime}-\widehat{J}\right)\right.$ intersects $\widehat{J}$ along a subcomplex of the collection of lines $\left.\left(q^{\prime}\right)^{-1}(A)\right)$, and hence it is finitely generated and free by the Grushko-Neumann theorem. Moreover, choosing base points on any given base ray, it is not hard to check that $\operatorname{rank}\left(\pi_{1}\left(\widehat{K}_{P}-\operatorname{int}\left(\widehat{C}_{i+1}\right)\right)\right) \geq \operatorname{rank}\left(\pi_{1}\left(\widehat{K}_{P}-\operatorname{int}\left(\widehat{C}_{i}\right)\right)\right)$ and the homomorphism $\pi_{1}\left(\widehat{K}_{P}-\operatorname{int}\left(\widehat{C}_{i+1}\right)\right) \longrightarrow \pi_{1}\left(\widehat{K}_{P}-\operatorname{int}\left(\widehat{C}_{i}\right)\right)$ can be taken to be a projection between finitely generated free groups.

\section{Appendix}

This section is intended to provided the background and notation needed in this paper, specially the notions of fundamental pro-group and semistability at infinity. In what follows, we will be working within the category tow $-G r$ of towers of groups whose objects are inverse sequences of groups

$$
\underline{A}=\left\{A_{0} \stackrel{\phi_{1}}{\longleftarrow} A_{1} \stackrel{\phi_{2}}{\longleftarrow} A_{2} \longleftarrow \cdots\right\}
$$

A morphism in this category will be called a pro-morphism. See [17, 28] for a general reference.

A tower $\underline{L}$ is a free tower if it is of the form

$$
\underline{L}=\left\{L_{0} \stackrel{i_{1}}{\longleftarrow} L_{1} \stackrel{i_{2}}{\longleftarrow} L_{2} \longleftarrow \cdots\right\}
$$

where $L_{i}=\left\langle B_{i}\right\rangle$ are free groups of basis $B_{i}$ such that $B_{i+1} \subset B_{i}$, the differences $B_{i}-B_{i+1}$ are finite and $\bigcap_{i=0}^{\infty} B_{i}=\emptyset$, and the bonding homomorphisms $i_{k}$ are given by the corresponding basis inclusions. On the other hand, a tower $\underline{P}$ is a telescopic tower if it is of the form

$$
\underline{P}=\left\{P_{0} \stackrel{p_{1}}{\longleftarrow} P_{1} \stackrel{p_{2}}{\longleftarrow} P_{2} \longleftarrow \cdots\right\}
$$

where $P_{i}=\left\langle D_{i}\right\rangle$ are free groups of basis $D_{i}$ such that $D_{i-1} \subset D_{i}$, the differences $D_{i}-D_{i-1}$ are finite (possibly empty), and the bonding homomorphisms $p_{k}$ are the obvious projections.

We will also use the full subcategory $(G r$, tow $-G r)$ of $M o r(t o w-G r)$ whose objects are arrows $\underline{A} \longrightarrow G$, where $\underline{A}$ is an object in tow $-G r$ and $G$ is a group regarded as a constant tower whose bonding maps are the identity. Morphisms in $(G r$, tow $-G r)$ will also be called pro-morphisms.

From now on, $X$ will be a (strongly) locally finite $\mathrm{CW}$-complex. A proper map $\omega:[0, \infty) \longrightarrow X$ is called a proper ray in $X$. We say that two proper rays $\omega, \omega^{\prime}$ define the same end if their restrictions $\left.\omega\right|_{\mathbb{N}},\left.\omega^{\prime}\right|_{\mathbb{N}}$ are properly homotopic. Moreover, we say that they define the same strong end if $\omega$ and $\omega^{\prime}$ are in fact properly homotopic. The $\mathrm{CW}$-complex $X$ is said to be semistable 
at infinity if any two proper rays defining the same end also define the same strong end.

Given a base ray $\omega$ in $X$ and a collection of finite subcomplexes $C_{1} \subset$ $C_{2} \subset \cdots \subset X$ so that $X=\bigcup_{n=1}^{\infty} C_{n}$, the following tower, pro $-\pi_{1}(X, \omega)$,

$$
\left\{\pi_{1}(X, \omega(0)) \leftarrow \pi_{1}\left(X-\operatorname{int}\left(C_{1}\right), \omega\left(t_{1}\right)\right) \leftarrow \pi_{1}\left(X-\operatorname{int}\left(C_{2}\right), \omega\left(t_{2}\right)\right) \leftarrow \cdots\right\}
$$

can be regarded as an object in $(G r$, tow $-G r)$ and it is called the fundamental pro-group of $(X, \omega)$, where $\omega\left(\left[t_{i}, \infty\right)\right) \subset X-\operatorname{int}\left(C_{i}\right)$ and the bonding homomorphisms are induced by the inclusions. This tower does not depend (up to pro-isomorphism) on the sequence of subcomplexes $\left\{C_{i}\right\}_{i}$. It is worth mentioning that if $\omega$ and $\omega^{\prime}$ define the same strong end, then pro $-\pi_{1}(X, \omega)$ and $\operatorname{pro}-\pi_{1}\left(X, \omega^{\prime}\right)$ are pro-isomorphic. In particular, we may always assume that $\omega$ is a cellular map. It is known that $X$ is semistable at infinity if and only if pro $-\pi_{1}(X, \omega)$ is pro-isomorphic to a tower where the bonding maps are surjections. Moreover, in this case $\pi_{1}^{e}(X, \omega)=\lim _{\longleftarrow}$ pro $-\pi_{1}(X, \omega)$ is a well-defined useful invariant which only depends (up to isomorphism) on the end determined by $\omega$ (see [19]). In a similar way, one can define objects in $(G r, t o w-G r)$ corresponding to the higher homotopy pro-groups of $(X, \omega)$.

Finally, given a finitely presented group $G$ and a finite 2-dimensional CW-complex $X$ with $\pi_{1}(X) \cong G$, we say that $G$ is semistable at infinity if the universal cover $\tilde{X}$ of $X$ is so, and we will refer to the fundamental pro-group of $\tilde{X}$ as the fundamental pro-group of $G$.

\section{References}

[1] Ayala, R., Cárdenas, M., Lasheras, F. F. and Quintero, A.: Properly 3-realizable groups. Proc. Amer. Math. Soc. 133 (2005), no. 5, 1527-1535.

[2] Brick, S. G. And Minalik, M.: The QSF property for groups and spaces. Math. Z. 220 (1995), 207-217.

[3] Brown, R. And Huebschmann, J.: Identities among relations. In Lowdimensional topology (Bangor, 1979), 153-202. London Math. Soc. Lecture Note Ser. 48. Cambridge Univ. Press, Cambridge-New York, 1982.

[4] Cárdenas, M., Fernández, T., Lasheras, F. F. and Quintero, A.: Embedding proper homotopy types. Colloq. Math. 95 (2003), no. 1, 1-20.

[5] Cárdenas, M. And Lasheras, F. F.: Properly 3-realizable groups: a survey. In Geometric methods in group theory, 1-9. Contemp. Math. 372. Amer. Math. Soc., Providence, RI, 2005.

[6] Cárdenas, M., Lasheras, F. F. and Quintero, A.: Proper homotopy invariants of properly 3 -realizable groups. Preprint.

[7] Cárdenas, M., Lasheras, F.F., Quintero, A. and Repovš, D.: Amalgamated products and properly 3-realizable groups. J. Pure Appl. Algebra 208 (2007), no. 1, 293-296. 
[8] Cárdenas, M., Lasheras, F.F. and Roy, R.: Direct products and properly 3-realisable groups. Bull. Austral. Math. Soc. 70 (2004), 199-205.

[9] Chiswell, I. M., Collins, D. J. and Huebschmann, J.: Aspherical group presentations. Math. Z. 178 (1981), 1-36.

[10] Cockcroft, W. H.: On two-dimensional aspherical complexes. Proc. London Math. Soc. (3) 4 (1954), 375-384.

[11] Dunwoody, M. J.: The accessibility of finitely presented groups. Invent. Math. 81 (1985), 449-457.

[12] Dyer, E. And Vasquez, A. T.: Some small aspherical spaces. Collection of articles dedicated to the memory of Hanna Neumann, III. J. Austral. Math. Soc. 16 (1973), 332-352.

[13] Funar, L., Lasheras, F. F. And Repovš, D.: Non-compact 3-manifolds proper homotopy equivalent to geometrically simply connected polyhedra and proper 3-realizability of groups. Preprint, arXiv:math/0709.1576.

[14] Fine, B. And Peluso, A.: Amalgam decomposition for one-relator groups. J. Pure Appl. Algebra 141 (1999), 1-11.

[15] Funar, L. And Gadgil, S.: On the geometric simple connectivity of open manifolds. Int. Math. Res. Not. 2004, no. 24, 1193-1248.

[16] Funar, L. And Otera, D. E.: Remarks on the WGSC and QSF tameness conditions for finitely presented groups. Preprint, arXiv:math/0610936.

[17] Geoghegan, R.: Topological methods in group theory. Graduate Texts in Mathematics 243. Springer, New York, 2008.

[18] Geoghegan, R. And Minalik, M.: Free abelian cohomology of groups and ends of universal covers. J. Pure and Appl. Algebra 36 (1985), 123-137.

[19] Geoghegan, R. and Minalik, M.: The fundamental group at infinity. Topology 35 (1996), no. 3, 655-669.

[20] Hog-Angeloni, C., Metzler, W. and Sieradski, A. J., editors: Twodimensional Homotopy and Combinatorial Group Theory. London Math. Soc. Lecture Notes Series 197. Cambridge Univ. Press, Cambridge, 1993.

[21] Karrass, A. And Solitar, D.: Subgroups of HNN groups and groups with one defining relation. Canad. J. Math. 23 (1971), 627-643.

[22] Lasheras, F. F.: Universal covers and 3-manifolds. J. Pure Appl. Algebra 151 (2000), no. 2, 163-172.

[23] Lasheras, F. F.: Ascending HNN-extensions and properly 3-realisable groups. Bull. Austral. Math. Soc. 72 (2005), 187-196.

[24] Lyndon, R. C.: Cohomology theory of groups with a single defining relation. Ann. of Math. (2) 52 (1950), 650-665.

[25] Lyndon, R. C. And Schupp, P. E.: Combinatorial group theory. Ergebnisse der Mathematik und ihrer Grenzgebiete, Band 89. Springer-Verlag, Berlin-New York, 1977.

[26] Magnus, W.: Über diskontinuierliche Gruppen mit einer definierenden Relation. (Der Freiheitssatz). J. Reine Angew. Math. 163 (1930), 141-165. 
[27] Magnus, W., Karrass, A. And Solitar, D.: Combinatorial group theory: Presentations of groups in terms of generators and relations. Interscience Publishers, New York-London-Sydney 1966.

[28] Mardešić, S. And Segal, J.: Shape Theory. The inverse system approach. North-Holland Mathematical Library 26. North-Holland, Amsterdam-New York, 1982.

[29] Mihalik, M.: Semistability at the end of a group extension. Trans. Amer. Math Soc. 277 (1983), no. 1, 307-321.

[30] Minalik, M. And Tschantz, S: One relator groups are semistable at infinity. Topology 31 (1992), no. 4, 801-804.

[31] Scott, P. And Wall, C. T. C.: Topological methods in group theory. In Homological group theory (Durham, 1977), 137-203. London Math. Soc. Lecture Note Ser. 36. Cambridge Univ. Press, Cambridge-New York, 1979.

Recibido: 21 de febrero de 2007

Revisado: 1 de octubre de 2007

Manuel Cárdenas

Departamento de Geometría y Topología

Universidad de Sevilla

Apdo 1160, 41080-Sevilla, Spain

mcard@us.es

Francisco F. Lasheras

Departamento de Geometría y Topología

Universidad de Sevilla

Apdo 1160, 41080-Sevilla, Spain

lasheras@us.es

Antonio Quintero

Departamento de Geometría y Topología

Universidad de Sevilla

Apdo 1160, 41080-Sevilla, Spain

quintero@us.es

Dušan Repovš

Institute of Mathematics, Physics and Mechanics

University of Ljubljana

P.O. Box 2964, Ljubljana 1001, Slovenia

dusan.repovs@fmf.uni-lj.si

The first three authors were supported by the project MTM 2007-65726. This research was also supported by Slovenian-Spanish research grant BI-ES/04-05-014. The authors acknowledge remarks and suggestions by Louis Funar. 